\title{
Fiberoptic endoscopic evaluation of the swallowing in neurogenic dysphagia: A proposal of an algorithm for fiberoptic endoscopic evaluation of the swallowing in neurology hospitals in Mexico
}

\author{
Ruben Jimenez-Dominguez ${ }^{1 *}$ and Carlos Manzano-Aquiahuat ${ }^{2}$ \\ ${ }^{1}$ Neurology Center, Vinzenz von Paul Hospital, Rottweil, Germany; ${ }^{2}$ Department of Phoniatrics, Hospital Médica Sur Centro Médico ABC, Mexico City, Mexico
}

\begin{abstract}
Fiberoptic endoscopic evaluation of swallowing (FEES) is an established and one of the most commonly used methods for assessing patients with oropharyngeal dysphagia. At least $50 \%$ of neurological patients, especially those with acute stroke, present with oropharyngeal dysphagia. These patients have a three-fold increased risk of pneumonia and poor outcome. FEES in oropharyngeal dysphagia is an important tool in early evaluation of neurological patients where dysphagia is suspected. FEES allows objectively evaluate deglutition, determine the risk of aspiration, detect silent aspirations, and implement initial therapeutic measures in patients with neurological disease. Our goal is to describe some basic concepts of FEES and propose an algorithm for the evaluation and assessment of patients with neurogenic oropharyngeal dysphagia in neurology hospitals and stroke centers in Mexico.
\end{abstract}

Key words: Fiberoptic endoscopic evaluation of the swallowing. Dysphagia. Oropharyngeal. Neurogenic. Stroke.

\section{Evaluación fibroendoscopica de la deglución (FEES) en disfagia neurogénica: propuesta de un algoritmo para la FEES en hospitales de neurología en México}

\section{Resumen}

La evaluación fibroendoscópica de la deglución (FEES) es un método bien establecido y uno de los más utilizados para la evaluación de pacientes con disfagia orofaríngea. Al menos el 50\% de los pacientes neurológicos, especialmente aquellos con enfermedad cerebrovascular presenta disfagia orofaríngea. Estos pacientes presentan tres veces mayor riesgo de neumonía y mal pronóstico. La FEES en la disfagia orofaríngea es una herramienta importante en la evaluación temprana del paciente neurológico con sospecha de disfagia. La FEES permite evaluar objetivamente la deglución, determinar el riesgo de aspiración, detectar aspiraciones silentes e implementar medidas terapéuticas tempranas en pacientes con enfermedad neurológica. Nuestro propósito es describir algunos aspectos básicos de la FEES y proponer un algoritmo para la evaluación del paciente con disfagia neurogénica orofaríngea en hospitales neurológicos y centros de ictus en México.

Palabras clave: Evaluación fibroendoscópica de la deglución (FEES). Disfagia. Orofaríngea. Neurogénica. Enfermedad cerebrovascular.

\section{Correspondence:}

*Ruben Jimenez-Dominguez

E-mail: r.jimenez@vvph.de

DOI: 10.24875/RMN.21000046
Available online: 01-10-2021 Rev Mex Neuroci. 2021;22(5):208-217 www.revmexneurociencia.com 2604-6180/ @ 2021 Academia Mexicana de Neurología A.C. Published by Permanyer. This is an open access article under the CC BY-NC-ND license (http://creativecommons.org/licenses/by-nc-nd/4.0/). 


\section{Introduction}

Dr. Susan Langmore developed the Fiberoptic Endoscopic Evaluation of Swallowing (FEES) procedure in $1988^{1}$. Due to technological advances and high-resolution imaging, we can now differentiate with clarity the anatomic structures of the pharynx and evaluate the act of deglutition. The evaluation of patients with dysphagia was led principally by laryngologists, phoniatricians, and speech language pathologists. Based on current needs, the German Society of Neurology, the German Stroke Society, and the German Society of Geriatrics have currently proposed a rigorous training curriculum for performing FEES in patients with neurogenic oropharyngeal dysphagia².

Braun et al. demonstrated the presence of dysphagia in $59.6 \%$ of neurological hospitalized patients ${ }^{3}$. In Mexico, Perez-Cruz et al. reported a prevalence of oropharyngeal dysphagia of $49.1 \%$ in ambulant neurological patients ${ }^{4}$.

Through clinical assessment and FEES, the goal is to detect patients with oropharyngeal dysphagia and prevent complications such as aspiration pneumonia in acute stroke patients in stroke units. Lindner-Pfleghar et al. proposed an algorithm for the evaluation and treatment of stroke patients, highlighting the use of FEES at a low threshold in patients with severe stroke and minor clinical symptoms, principally by isolated dysarthria, and cough after swallowing ${ }^{5}$. These two variables were identified as independent predictors of aspiration.

We propose an algorithm for the assessment and detection of neurogenic dysphagia in hospitalized neurological patients in Mexico, with an emphasis on stroke patients, based on the recommendations of Dr. Langmore and the European Society for Swallowing Disorders.

\section{Basic anatomic and functional concepts in deglutition}

Deglutition or swallowing is a complex process that is defined as the passing and transport of different materials from the oral cavity to the stomach. For academic and practical purposes, we divided the process of swallowing in four stages:

1. Oral preparatory phase: In this phase, the food or fluids are introduced into the mouth and transported to the anterior portion of the tongue. The lips close and the mastication process begins by rotation movements of the tongue. The descending of the velum prevents the pass of the bolus or parts of it to the oropharynx. In some patients, by eating mixed consistencies, fluids can pass during chewing into the oropharynx due to the absence of the tongue-velum closure. This causes a risk of aspiration.

2. Oral transport phase: In this phase, the Bolus is transported into the oropharynx.

3. Pharyngeal phase: The pharyngeal phase begins when the bolus reaches the palatoglossal arch and triggers the swallowing reflex. The pharyngeal phase protects the airway by swallowing apnea and interrupting the expiratory phase of breathing, lasting approximately $0.5-1.5 \mathrm{~s}$. The cuneiform cartilage moves to anteriorly, the epiglottis descends and the vocal cords close, which helps direct the food bolus toward the piriform fossa and into the esophagus by opening the upper esophageal sphincter. The onset of the pharyngeal phase can be observed with the endoscopy. A period of whiteout can be appreciated with the overlapping of the swallowing events.

4. Esophageal phase: During this phase, the upper esophageal sphincter closes. With peristaltic pressure of the esophagus the food bolus enters into the stomach.

\section{The central control of swallowing}

Many structures of the central nervous system such as the somatosensory cortex, supplementary motor area, operculum, prefrontal and inferior frontal cortex, cingulate cortex, insular cortex, thalamus, basal ganglia, cerebellum, pons, and medulla are involved in the controlling swallowing. The activation of these brain structures depends on the initial stimuli that trigger swallowing. Leopold and Daniels described a neuroanatomical model of swallowing control, in which all the aforementioned brain regions formed a complex neuronal network ${ }^{6}$.

The dominance of the cortical control of swallowing varies among individuals. The preparatory and oral transport phases are controlled predominantly in the left hemisphere, while the right hemisphere predominantly controls the pharyngeal phase ${ }^{7}$.

The corticobular tract coordinates input and output to and from the primary somatosensory cortex to the pontomedullary swallowing center. Lesions in this region cause an alteration in the coordination of swallowing movements?

A lesion in the posterior part of the parietal and temporal lobes causes oropharyngal residues, a delay in the swallowing responses, and an impaired laryngeal vestibular closure $^{8}$. The insula contributes to the timing and synchronization of the swallowing muscles; thus, 
insular lesions cause a delay in the initiation of pharyngeal swallowing. Lesions of the thalamus provoke an alteration in hyoid movement. Furthermore, the basal ganglia and the thalamus play an important role in the sensory input during deglutition. The ponto-medullary swallowing center is a group of interneurons consisting of the dorsal and ventral swallowing groups, located in the lateral portion of the pontomedullary junction. These neurons play an important role in the organization, sensible innervation, and motor control through the cranial nerves V, VII, IX, X, and XII.

\section{Clinical indications for FEES}

The indications for FEES are variable, including logistic or clinical reasons, compromised pulmonary clearance, evaluation of secretions management, positioning problematic, and unavailable fluoroscopy, among others. We should consider performing FEES in any patient suspected of dysphagia in the clinical screening tests, or where there is the presence of severe dysarthria, facial palsy, and aphasia. Neurological diseases related to dysphagia include primary acute stroke, Parkinson's disease, neuromuscular disease, motoneuron disease with bulbar affection, and acute inflammatory demyelinating Polyneuropathy.

\section{Screening of dysphagia}

We recommend any validated standard screening for the initial patient evaluation, such as, the volume-viscosity swallow test (V-VST), the Standardized Swallowing Assessment (SSA), the $90 \mathrm{ml}$ screening test, and the 2- 6 scale by Daniels, among others ${ }^{9-14}$. These tests could be performed by any hospital care provider including nursing staff, speech therapists, physiotherapists, and physicians.

The authors recommend the pragmatic use, for example, the V-VST as a bedside screening method because of its high psychometric features and, easy feasibility. By evaluating different viscosities, V-VST allows us to implement the initial therapeutic measures regarding oral intake. This article does not describe in detail the performing of the screening tests. For a detailed revision, we recommend reading the proposal of Mercedes Velasco et al. for the clinical evaluation of dysphagia ${ }^{9}$.

\section{FEES}

After explaining and preparing the patient for the FEES procedure, we recommend the use of a local

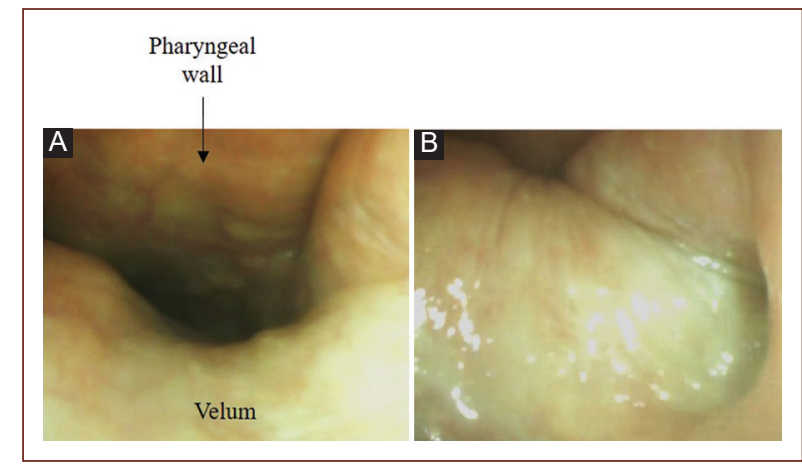

Figure 1. A: relaxed position of the velum B: elevation of the velum during speech.

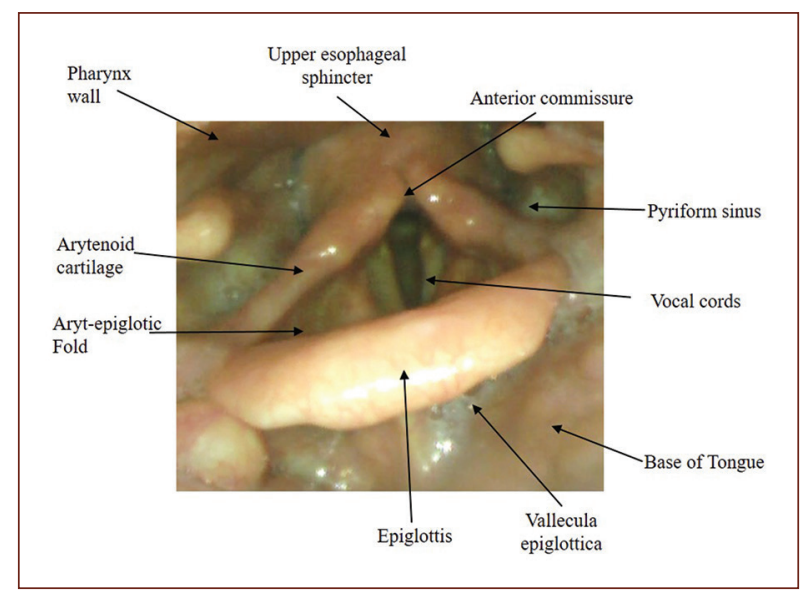

Figure 2. Home position, anatomical structures of the oropharynx.

anesthetic in the nasal cavity. The patient must be examined in a sitting position. The endoscope is put into the nasal cavity and must be directed in a caudal and posterior direction.

We recommend the following steps for the systematic FEES.

1. Ask the patient to say explosive and ficative words such as "coco, cuca come queso" and observe the elevation of the velum (Fig. 1).

2. Home position, oropharyngeal view (Fig. 2):

- Observation of the anatomical structures of the oropharynx

- Symmetry.

- Ventilation and phonation positions.

- Evaluation of the phonetics and closure of the vocal cords:

- Ask the patient to say "iiiii" for 2-3 s, then from deep to acute tone. Depending on the mobility of 
the vocal cords, the use of different phonatory tasks may be needed.

- Ask the patient to cough and to clear the throat. Perform the Valsalva maneuver, and observe closure of the vocal cords.

- Perform pharyngeal squeeze maneuver: The pharyngeal squeeze maneuver is a validated measure of pharyngeal strength and evaluates the pharyngeal motor integrity. The patient is asked to make an "eeeee"-Phonation, the hypopharyngeal lumen constricts by bilateral contraction of the pharynx muscles.

- Evaluate sensibility by touching the hypopharyngeal structures with the top of the endoscope, this can however be uncomfortable for the patient and trigger a protective reaction such as coughing, sneezing, or throat clearing.

- Identification of signs of impaired swallowing function and sensibility

- Reduction of swallowing frequency.

- Secretions and alimentary residues in base of tongue, vallecula, piriform sinuses, retropharyngeal space, and laryngeal vestibule.

- Penetration/aspiration.

3. Observation of swallowing process with different food consistencies:

- Pureed and mashed fruits and vegetables.

- Thickened water (e.g. nectar): It is important to evaluate swallowing with thickened water to have a founded recommendation on the changes in diet in the presence of dysphagia. We cannot recommend the use of thickened water without objectifying its effects.

- Fluids: It is important to first give the patient one to two Teaspoons of fluid, and more if possible, with a glass. In our experience, the use of milk or vegetal color (green dye) allows to more accurately observe the pathological findings during swallowing, such as penetration and aspiration.

- Foods with solid consistency (e.g. toasted bread and banana).

4. Perform restitution maneuvers such as Masako-Maneuver (tongue hold), chin tuck (drop of the chin to or toward the chest), and rotation of the head ipsi- and contralateral to the affected side ${ }^{15,16}$.

After performing FEES, we recommend a protocol to describe the pathologic findings of the swallowing phases, using scales to describe the grade of secretions and residues, as well as the presence of penetration and aspiration. In summary, we recommend a pathophysiological description of the affected swallowing phases, with an emphasis on aspiration risk. Finally, recommendations for therapy should be described. At this point, the intervention of the laryngologists/phoniatrician/speech language pathologist is important to determine the therapeutic considerations. The Murray secretion scale, Yale pharyngeal residue severity rating scale, and penetration aspiration scale (PAS) are validated instruments that allow to object our FEES-findings and improve the accuracy of the interpretation.

We calibrate the presence of secretion according to the Murray scale as follows.

Simplified Murray secretion scale ${ }^{17}$.

- 0 - Normal (no secretions)

- 1 - Pooling in vallecuale/pyriform sinus

- 2 - Pooling in laryngeal vestibule transiently

- 3 - Poolling in laryngeal vesibule consistently

The Yale Pharyngeal Residue Severity Rating Scale is a five-point rating scale for the assessment of post-swallow pharyngeal residue severity in FEES. The severity of the residue must be described separately in the epiglottic vallecula and pyriform sinus as follows.

Yale Pharyngeal Residue Severity Rating Scale ${ }^{18,19}$. $0 \%$ (none): Vallecula/pyriform sinus- no residue.

$1-5 \%$ (trace): Vallecula/pyriform sinus- trace coating of mucosa.

5-25\% (mild): Vallecula -epiglottic ligament visible.

Pyriform sinus- up wall to quarter full.

$25-50 \%$ (moderate): Vallecula- epiglottic ligament cover.

Pyriform sinus- up wall to half full.

$>50 \%$ (severe): Vallecula- filled to epiglottic rim.

Pyriform sinus- filled to aryepiglottic fold.

The PAS is an 8-point rating scale that assesses the presence of penetration and/or aspiration as follows 20 :

1 (None) - No entry of material into the larynx or trachea.

2 (penetration) - Entry of material into the larynx with clearing.

3 (penetration) - Entry of material into the larynx without clearing.

4 (penetration) - Material contacts the true vocal folds with clearing.

5 (penetration) - Material contacts the true vocal folds without clearing.

6 (aspiration) - Material enters the trachea and is sponaeously cleared into the larynx or pharynx.

7 (aspiration) - Material enters the trachea and is not cleared following attempts. 
Table 1. Fiberoptic endoscopic dysphagia severity scale (FEDSS) ${ }^{20,21}$

\begin{tabular}{|c|c|c|c|}
\hline Score & FEDSS & Findings & Recommendations \\
\hline 6 & Saliva & Penetration/Aspiration & $\begin{array}{l}\text { Non per os, gastric tube, protective } \\
\text { endotracheal intubation }\end{array}$ \\
\hline 5 & Soft bite/easy to chew food consistency & $\begin{array}{l}\text { Penetration/Aspiration with } \\
\text { reduced or absence of protective } \\
\text { reflexes }\end{array}$ & Non per os, gastric tube \\
\hline 4 & Soft bite/easy to chew food consistency & $\begin{array}{l}\text { Penetration/Aspiration with normal } \\
\text { protective reflexes }\end{array}$ & Logopedic therapy, eventually gastric tube \\
\hline 4 & Fluid & $\begin{array}{l}\text { Penetration/Aspiration with } \\
\text { reduced or absence of protective } \\
\text { reflexes }\end{array}$ & Logopedic therapy, eventually gastric tube \\
\hline 3 & Fluid & $\begin{array}{l}\text { Penetration/Aspiration with normal } \\
\text { protective reflexes }\end{array}$ & $\begin{array}{l}\text { Logopedic therapy Dysphagia adapted } \\
\text { Diet, eventually intravenous fluids }\end{array}$ \\
\hline 2 & Hard/dry food consistency & $\begin{array}{l}\text { Penetration/Aspiration, severe } \\
\text { residues }\end{array}$ & $\begin{array}{l}\text { Logopedic therapy Dysphagia adapted } \\
\text { Diet, oral fluids }\end{array}$ \\
\hline 1 & Hard/dry food consistency & $\begin{array}{l}\text { No penetration/aspiration, no } \\
\text { relevant residues }\end{array}$ & Regular or transitional foods \\
\hline
\end{tabular}

8 (aspiration) - Material enters the trachea with no attempt to clear.

The PAS is a descriptive tool with categorical characteristics that is helpful to understand the pathophysiological phenomena that occur during swallowing. Some limitations of the PAS are that it only describes the depth of airway invasion and clearing, and the lack of discrimination between possible-aspiration mechanisms. The entry of material into the larynx with clearing (PAS 2) is a phenomenon that also occurs in healthy individuals and should be considered abnormal only in the context with the clinical and other pathological findings in FEES. Penetration and aspiration can sometimes be challenging to see, because swallowing is dynamic, which is a process is seen in real time. Thus, it is important the FEES-Devices have a high quality of temporal resolution. Another limitation of this scale includes discrepancies in the statistical use.

\section{Evaluation of the severity of dysphagia}

The Fiberoptic Endoscopic Dysphagia Severity Scale (FEDSS, Table 1) independently predicts the outcome and complications in the early evaluation of acute stroke patients. Each increase of 1 point conferred a more than two-fold increased risk of developing pneumonia ${ }^{21}$.
Table 2. Simplified dysphagia severity rating scale (DSRS) $)^{2,22}$

\begin{tabular}{|l|l|l|l|}
\hline Score & & Diet & Supervision \\
\hline 4 & $\begin{array}{l}\text { No oral } \\
\text { fluids }\end{array}$ & Non per os & No oral feeding \\
\hline 3 & $\begin{array}{l}\text { Pudding } \\
\text { consistency }\end{array}$ & Puree & Therapeutic feeding \\
\hline 2 & $\begin{array}{l}\text { Custard } \\
\text { consistency }\end{array}$ & $\begin{array}{l}\text { Soft, moist } \\
\text { diet }\end{array}$ & $\begin{array}{l}\text { Feeding by third } \\
\text { party (untrained) }\end{array}$ \\
\hline 1 & $\begin{array}{l}\text { Syrup } \\
\text { consistency }\end{array}$ & $\begin{array}{l}\text { Selected } \\
\text { textures }\end{array}$ & $\begin{array}{l}\text { Eating with } \\
\text { supervision }\end{array}$ \\
\hline 0 & Normal & Normal & Eating independently \\
\hline
\end{tabular}

The Dysphagia Severity Rating Scale (Table 2) is based on oral intake and supervision requirements, and is also a useful and validated tool for the severity assessment of post-stroke patients ${ }^{22}$. Thus, any validated scale must be a tool for determining therapeutical consequences. The authors recommend both scales to assess of the severity of neurogenic oropharyngeal dysphagia.

\section{Basic concepts for the interpretation of FEES}

A delay of the swallowing reflex occurs when a fluid bolus reaches the vallecula and the patient swallows 
after $3 \mathrm{~s}$. With dry food consistencies, the swallowing reflex should occur in a maximum of $2 \mathrm{~s}$.

Leaking is an uncontrolled premature escape of parts of the bolus into the pharynx.

A delay of the trigger of the swallowing reflex occurs as a consequence of a reduction in sensibility.

Penetration is the passage or presence of materials in the laryngeal Aditus above the vocal cords (Fig. 3).

Aspiration is defined as the passage of materials through the vocal cords in the subglotic region (Trachea) (Fig. 3).

Silent penetration/aspiration occurs without activation of protective mechanisms, such as Coughing and throat-clearing.

Retention is the remnants of bolus material in the Hypopharynx nach swallow.

Hypersalivation and poor clearing occurs in patients with reduced sensibility or also seen in patients with reflux, in this case, a foamy secretion is seen.

We propose the following algorithm for the evaluation of neurogenic dysphagia in a hospital setting (Fig. 4) and a protocol for the FEES description (Table 3). A systematic description allows detection and a wellbased interpretation of pathological findings.

\section{The FEES-tensilon test}

FEES in the diagnosis of neuromuscular disease is an important tool that allows identification and to objectification of pathological findings in patients with predominantly bulbar symptoms, particularly under suspect of myasthenia gravis. We perform FEES in combination with the Tensilon test as a diagnostic test. The FEES-Tensilon test must be performed based on standard protocol and by giving 30 consecutive white broad pieces (fatigable swallowing test) ${ }^{23}$. After 5-10 swallows more residues can be observed.

\section{Discussion}

Lindner-Pfleghar et al. ${ }^{5}$ discussed some discrepancies in the clinical assessment tools SSA and 2 to 6 with the FEES findings regarding silent aspirations. The FEES test is a relatively simple method to perform and well tolerated by patients, and can reveal silent aspirations in patients with no clinical evidence. FEES is a safe procedure with few and rare complications such as epistaxis and transient bradycardia ${ }^{24}$. Nacci et al. evaluated the safety of the FEES method and described an incidence of laryngospasm of $0.07 \%$, related to the presence of neuromuscular diseases ${ }^{25}$. FEES seems

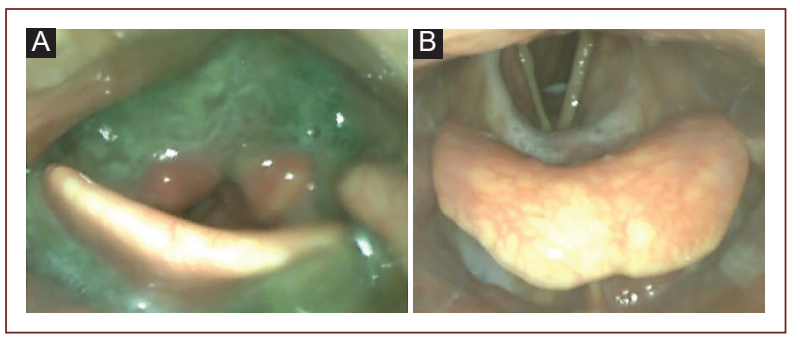

Figure 3. Pathological findings. A: severe residues in the vallecula epiglottis and pyriform sinus, note the presence of thickened material in the interarytenoid region with penetration/aspiration risk. B: penetration and aspiration of fluid without the trigger of protective reflexes.

to be a safe method for the assessing the swallowing function even in acute stroke patients ${ }^{26}$.

Although Videofluoroscopy (VFSS) is considered the gold standard method for the evaluation of dysphagia, the FEES-test offers more accessibility for evaluation of acute bedside patients with immobility and/or altered consciousness in stroke units. FEES uses real food and allows a better view of larynx movement ${ }^{27}$. It is particularly important in evaluating the pharyngeal phase of deglutition, visualizing secretions, penetrations, and assessing the efficacy of the protective mechanisms and sensibility of the pharyngeal structures. This method is sensitive to the visualization of silent aspiration and is especially useful for evaluation of post-swallowing residues.

Unlike VFSS and oro-pharingo esophageal scintigraphy (OPES), the FEES method does not expose the patient to radiation; thus, it can be repeated several times for accurate follow-up of dysphagia ${ }^{28}$. Videofluoroscopy evaluates all deglutition phases such as the function of the upper esophageal sphincter.

The efficacy and practicability of the FEES method are well demonstrated. Regarding clinical outcome and influence in the incidence of pneumonia, both methods have not shown significant difference ${ }^{29}$. However, follow-up and long-term outcome are determined by many variables including the presence of risk factors, comorbidity, and the degree of disability among others. Both methods are practical tools for the evaluation of swallowing, which allows us to take pertinent therapeutic measures. In our experience, FEES is more accessible to the clinician in a hospital setting, especially in the early evaluation of hospitalized patients during the acute phase. After clinical evaluation of an acute neurological patient on hospital admission with an emphasis on the stroke patient, FEES should be performed at bedside to 


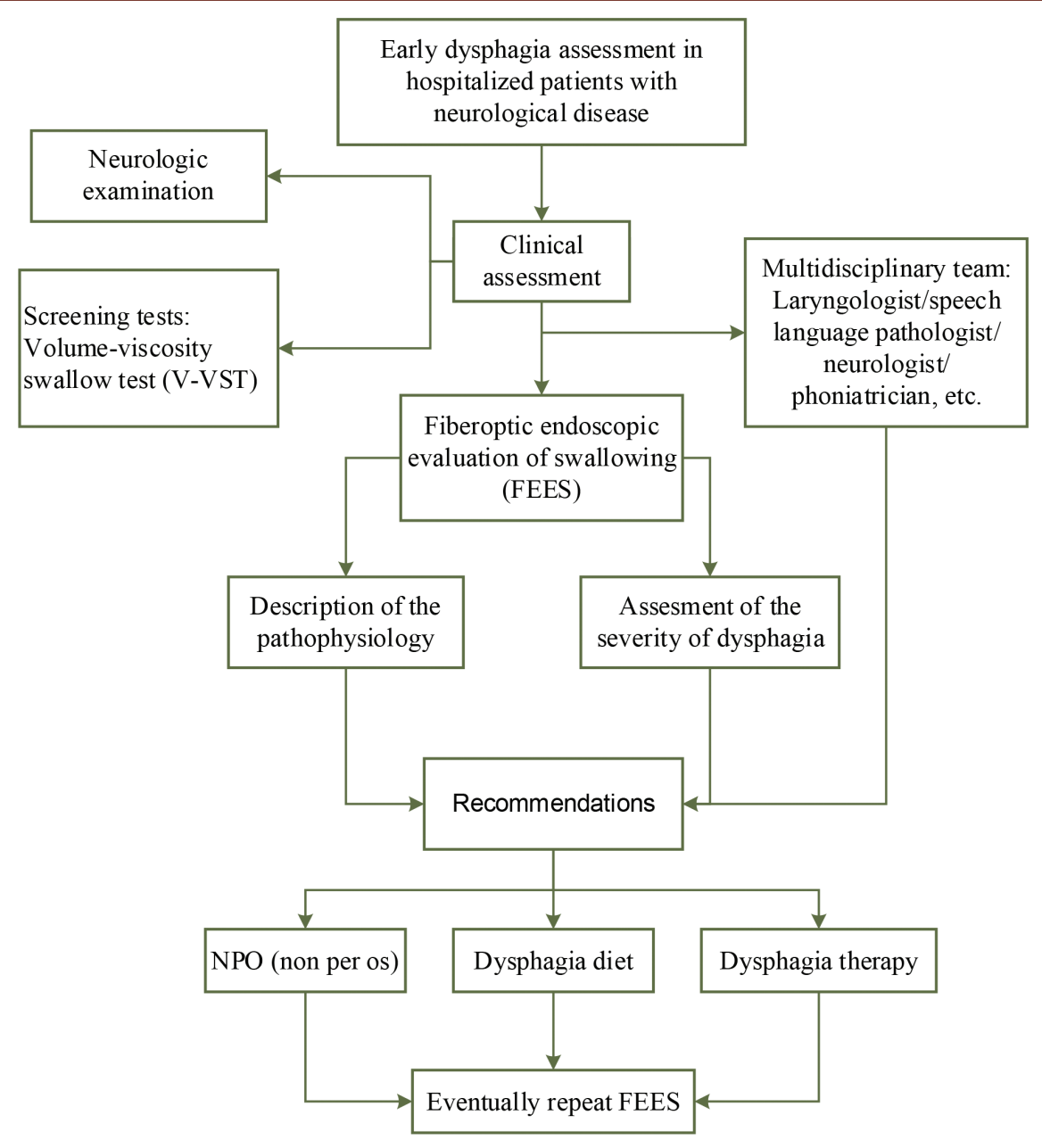

Figure 4. Algorithm for the evaluation of the patient with oropharyngeal neurogenic dysphagia.

make a prompt therapeutic decision regarding oral intake and diet modification. We recommend performing FEES within the first 24-48 $\mathrm{h}$ depending on the severity of symptoms and presence of clinical predictors of dysphagia. A follow-up could be performed considering the clinical outcome during the early rehabilitation phase, especially in patients with tracheostomy tubes, to allow an earlier resumption of oral feeding and implementation of a symptom-oriented rehabilitation of dysphagia. Early rehabilitation in the acute phase of stroke improves clinical outcome.

The findings of the VFSS and FEES are complementary methods, which allow the clinician to perform adequate intervention in the acute phase and a devise a therapeutical strategy for the rehabilitation of deglutition.

As mentioned earlier, the FEDSS evaluates the severity of dysphagia in acute stroke patients and rapidly guides the clinician in decision-making regarding therapeutic measures. Insertion of a gastric tube should be considered in patients with penetration/aspiration of saliva, soft bite consistencies, or fluids, without effective protective reflexes. Permanent aspiration of saliva, secretions, regurgitation of stomach content without cough reflex, ineffective voluntary cough, and repetitive pneumonia, are indications for tracheotomy. For fluid aspiration/penetration, the use of thickened fluids is a good alternative, effectivity of which must be objectified with FEES, before a recommendation is made. FEES plays an important role in the decision of diet therapy based on endoscopic observations and patient symptoms. After the acute phase of the disease, FEES should be performed according to the evolution of the patient during dysphagia therapy. We recommend the use of FEES in the early evaluation and following the acute phase, when the patient is more stable. The 
Table 3. Proposal for a descriptive FEES-protocol

Date:

Patient's name:

Diagnosis:

Trans nasal access: right/left

Observation before swallowing

\begin{tabular}{|l|l|l|l|}
\hline & Normal & Description & Pathological findings \\
\hline
\end{tabular}

Velopharyngeal closure

Glotis closure

Vocal cords closure

Pharynx contraction

Mucosa

Interarytenoid region

Phonation

Cough

Abdominal push

Opening of the vocal cords during sniffing

Murray secretion scale

\begin{tabular}{|l|l|l|l|}
\hline \multicolumn{4}{|c|}{ Observation during swallowing } \\
\hline Food consistency & Normal & Description & Pathological findings \\
\hline
\end{tabular}

Quantity:

\section{Predeglutitive:}

Oral preparation

Oro-pharyngeal food passing

Bolus control

Triggering of the swallow reflex

Penetration

Aspiration

\section{Intradeglutitive: \\ White-out}

\section{Postdeglutitive:}

Residues

Penetration

Spontaneous clearing

Yale retention score

Compensatory techniques

Vallecula epiglotica:

Pyriform sinus:

\begin{tabular}{|l|l|l|l|l|l|}
\hline \multicolumn{5}{|c|}{ Scales/Scores } \\
\hline Food consistency & PAS & Yale-Residue & FEDSS & DSRS & Other scales \\
\hline Fluids & & & \\
\hline Thickened water & & & \\
\hline Pureed & & & \\
\hline Dry
\end{tabular}


Table 3. Proposal for a descriptive FEES-protocol (Continued)

\begin{tabular}{|c|c|c|}
\hline Date: & & \\
\hline Patient's name: & & \\
\hline Diagnosis: & & \\
\hline Trans nasal access & & \\
\hline & Observatio & fore swallowing \\
\hline Summary assesme & & \\
\hline Food consistency & & \\
\hline Regular & & \\
\hline Soft/Bite-sized & Alone & With supervision \\
\hline Minced and Moist & Alone & With supervision \\
\hline Pureed & Alone & With supervision \\
\hline Fluids & Thickened & Normal \\
\hline Non per os & & \\
\hline
\end{tabular}

FEES-findings will help us modify the dysphagia therapy and diet recommendations.

Thus, the evaluation and treatment of oropharyngeal dysphagia must be considered as an interdisciplinary approach.

\section{Conclusion}

FEES has gained acceptance in assessing of neurological patients suspected of oropharyngeal dysphagia. It is important to consider the four predictors of aspiration for every patient screening (dysarthria, dysphonia, cough after swallowing of water, and abnormal cough reflex) and perform FEES in any patient with clinically suspect of dysphagia, to detect silent aspirations. The use of scales is an important tool in the interpretation and detection of the risk of aspiration; however, it is sometimes confusing. Therefore, we recommend parallel use of scales to describe the pathological findings according to the deglutition phases. The description of pathological findings allows us to understand possible pathophysiological mechanisms of neurological disease. Future studies should be conducted to validate an adaptation of the international severity scales in acute stroke patients in Mexican hospitals.

Endoscope devices for FEES are not always available in an hospital setting. Dysphagia frequently presents in neurological patients and increases the risk of pneumonia and mortality, particularly in stroke patients. Therefore, it is important to improve the management of dysphagia in all neurological hospitals, where acute stroke patients are treated ${ }^{30}$. We recommend continuing education in all the disciplines that address neurological dysphagia, supported by the several medical academies, to maintain high-quality standards, prevent complications such as aspiration, reduce hospital stays, and costs while improving the clinical outcome.

\section{Acknowledgments}

We thank Dr. Antonio Arauz for his comments and contributions to this publication.

\section{Funding}

There was no financial supporting.

\section{Conflicts of interest}

The authors declare no conflict of interest.

The Spanish version of the figures and tables is available upon the reader's request through the corresponding author.

\section{Ethical responsibilities}

Protection of human and animal subjects. The authors declare that no experiments were performed on humans or animals for this study. 
Confidentiality of data. The authors declare that no patient data appear in this article.

Right to privacy and informed consent. The authors declare that no patient data appear in this article.

\section{References}

1. Langmore SE, Schatz K, Olsen N. Fiberoptic endoscopic examination of swallowing safety: a new procedure. Dysphagia. 1988;2:216-9.

2. Dziewas R, Glahn J, Helfer C. FEES für neurogene dysphagien. Nervenarzt. 2014;85:1006-15.

3. Braun T, Juenemann M, Viard M. What is the value of fibre-endoscopic evaluation of swallowing (FEES) in neurological patients? A cross-sectional hospital-based registry study. BMJ Open. 2018;8:e019016.

4. Pérez-Cruz E, González-Muñoz A, Barrientos-Jiménez M, Camacho-Guerra CD, Tapia-Gómez Y, Torres-González KO, et al. Evaluación de la disfagia en pacientes con enfermedades neurológicas y su relación con riesgo de desnutrición. Med Intern México. 2018;34:359-65.

5. Lindner-Pfleghar B, Neugebauer H, Stösser S, Kassubek J, Ludolph A, Dziewas R, et al. Dysphagiemanagement beim akuten Schlaganfall: eine prospektive Studie zur Überprüfung der geltenden Empfehlungen. [Management of dysphagia in acute stroke: a prospective study for validation of current recommendations]. Nervenarzt. 2017;88:1739.

6. Leopold NA, Daniels SK. Supranuclear control of swallowing. Dysphagia. 2010;25:250-7.

7. Mihai PG, Otto M, Platz T, Eickhoff SB, Lotze M. Sequential evolution of cortical activity and effective connectivity of swallowing using fMRI. Hum Brain Mapp. 2014;35:5962-73.

8. Wilmskoetter J, Daniels SK, Miller AJ. Cortical and subcortical control of swallowing-can we use information from lesion locations to improve diagnosis and treatment for patients with stroke? Am J Speech Lang Pathol. 2020;29:1030-43

9. Velasco MM, Arreaola V, Clavé P, Puiggrós C. Abordaje clinico de la disfagia orofaríngea: diagnóstico y tratamiento. Nutr Clín Med. 2007; 1:174-202.

10. Correa-Flores M, Arch-Tirado E, Villeda-Miranda A. Análisis de la disfagia orofaríngea por medio de la evaluación fibroendoscópica de la deglución en pacientes con enfermedad de Parkinson. Cir Cir. 2012;80:31-7.

11. Perry $L$. Screening swallowing function of patients with acute stroke. Part one: identification, implementation and initial evaluation of a screening tool for use by nurses. J Clin Nurs. 2001;10:463-73.

12. Prosiegel M, Riecker A, Weinert M. Erratum zu: dysphagie-management in der akuten schlaganfallphase. Nervenarzt. 2014;84:226-8.
13. Jiang JL, Fu SY, Wang WH, Ma YC. Validity and reliability of swallowing screening tools used by nurses for dysphagia: a systematic review. $\mathrm{Ci} \mathrm{Ji}$ Yi Xue Za Zhi. 2016;28:41-8.

14. Daniels SK, McAdam CP, Brailey K. Clinical assessment of swallowing and prediction of dysphagia severity. Am J Speech Lang Pathol. 1997;6:17-24.

15. Saconato M, Chiari BM, Lederman HM, Gonçalves MI. Effectiveness of chin-tuck maneuver to facilitate swallowing in neurologic dysphagia. Int Arch Otorhinolaryngol. 2016;20:13-7.

16. Ertekin C, Keskin A, Kiylioglu N, Kirazli Y, On AY, Tarlaci S, et al. The effect of head and neck positions on oropharyngeal swallowing: a clinical and electrophysiologic study. Arch Phys Med Rehabil. 2001;82:1255-60.

17. Kuo CW, Allen CT, Huang CC. Murray secretion scale and fiberoptic endoscopic evaluation of swallowing in predicting aspiration in dysphagic patients. Eur Arch Otorhinolaryngol. 2017;274:2513-9.

18. Neubauer PD, Rademaker AW, Leder SB. The yale pharyngeal residue severity rating scale: an anatomically defined and image-based tool. Dysphagia. 2015;30:521-8.

19. Neubauer PD, Hersey DP, Leder SB. Pharyngeal residue severity rating scales based on fiberoptic endoscopic evaluation of swallowing: a systematic review. Dysphagia. 2016;31:352-9.

20. Rosenbek JC, Robbins JA, Roecker EB, Coyle JL, Wood JL. A penetration-aspiration scale. Dysphagia. 1996;11:93-8.

21. Warnecke T, Ritter MA, Kroger B, Oelenberg S, Teismann I, Heuschmann PU, et al. Fiberoptic endoscopic dysphagia severity scale predicts outcome after acute stroke. Cerebrovasc Dis. 2009;28:283-9.

22. Everton LF, Benfield JK, Hedstrom A. Psychometric assessment and validation of the dysphagia severity rating scale in stroke patients. Sci Rep. 2020;10:7268.

23. Warnecke T, Teismann I, Zimmermann J, Oelenberg S, Ringelstein EB, Dziewas R. Fiberoptic endoscopic evaluation of swallowing with simultaneous Tensilon application in diagnosis and therapy of myasthenia gravis. J Neurol. 2008;255:224-30.

24. Dziewas R, Auf dem Brinke M, Birkmann U, Bräuer G, Busch K, Cerra F, et al. Safety and clinical impact of FEES results of the FEES-registry. Neurol Res Pract. 2019;1:16.

25. Nacci A. Complications with fiberoptic endoscopic evaluation of swallowing in 2,820 examinations. Folia Phoniatr Logop. 2016;68:37-45

26. Warnecke $T$. The safety of fiberoptic endoscopic evaluation of swallowing in acute stroke patients. Stroke. 2009;40:482-6.

27. Kelly AM, Leslie P, Beale T. Fiberoptic endoscopic evaluation of swallowing and videofluoroscopy: does examination type influence perception of pharyngeal residue severity? Clin Otolaryngol. 2006;31:425-32.

28. Fattori B, Giusti P, Mancini V, Grosso M, Barillari MR, Bastiani L, et al. Comparison between videofluoroscopy, fiberoptic endoscopy and scintigraphy for diagnosis of oro-pharyngeal dysphagia. Acta Otorhinolaryngol Ital. 2016;36:395-402.

29. Aviv JE. Prospective, randomized outcome study of endoscopy versus modified barium swallow in patients with dysphagia. Laryngoscope. 2000;110:563-74.

30. Feng MC, Lin YC, Chang YH, Chen CH, Chiang HC, Huang LC, et al. The mortality and the risk of aspiration pneumonia related with dysphagia in stroke patients. J Stroke Cerebrovasc Dis. 2019;28:1381-7. 